$c 13.44: 64$

NBS MONOGRAPH 64

Refractive Indices and Densities of Aqueous Solutions of Invert Sugar

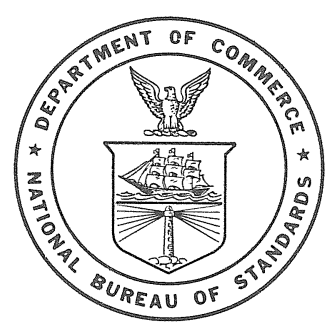

U.S. DEPARTMENT OF COMMERCE NATIONAL BUREAU OF STANDARDS 


\section{THE NATIONAL BUREAU OF STANDARDS}

\section{Functions and Activities}

The functions of the National Bureau of Standards are set forth in the Act of Congress, March 3, 1901, as amended by Congress in Public Law 619, 1950. These include the development and maintenance of the national standards of measurement and the provision of means and methods for making measurements consistent with these standards; the determination of physical constants and properties of materials; the development of methods and instruments for testing materials, devices, and structures; advisory services to government agencies on scientific and technical problems; invention and development of devices to serve special needs of the Government; and the development of standard practices, codes, and specifications. The work includes basic and applied research, development, engineering, instrumentation, testing, evaluation, calibration services, and various consultation and information services. Research projects are also performed for other government agencies when the work relates to and supplements the basic program of the Bureau or when the Bureau's unique competence is required. The scope of activities is suggested by the listing of divisions and sections on the inside of the back cover.

\section{Publications}

The results of the Bureau's research are published either in the Bureau's own series of publications or in the journals of professional and scientific societies. The Bureau itself publishes three periodicals available from the Government Printing Office: The Journal of Research, published in four separate sections, presents complete scientific and technical papers; the Technical News Bulletin presents summary and preliminary reports on work in progress; and Central Radio Propagation Laboratory Ionospheric Predictions provides data for determining the best frequencies to use for radio communications throughout the world. There are also five series of nonperiodical publications: Monographs, Applied Mathematics Series, Handbooks, Miscellaneous Publications, and Technical Notes.

A complete listing of the Bureau's publications can be found in National Bureau of Standards Circular 460, Publications of the National Bureau of Standards, 1901 to June 1947 (\$1.25), and the Supplement to National Bureau of Standards Circular 460, July 1947 to June 1957 (\$1.50), and Miscellaneous Publication 240, July 1957 to June 1960 (includes Titles of Papers Published in Outside Journals 1950 to 1959) (\$2.25); available from the Superintendent of Documents, Government Printing Office, Washington 25, D.C. 
UNITED STATES DEPARTMENT OF COMMERCE • Luther H. Hodges, Secretary NATIONAL BUREAU OF STANDARDS • A. V. Astin, Director

\title{
Refractive Indices and Densities of Aqueous Solutions of Invert Sugar
}

\author{
Carl F. Snyder and Albert T. Hattenburg
}

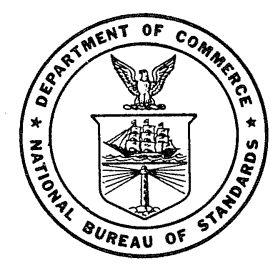

National Bureau of Standards Monograph 64

Issued June 7, 1963

For sale by the Superintendent of Documents, U.S. Government Printing Office 


\section{Contents}

1. Refractive index at various temperatures.

1. 1. Introduction

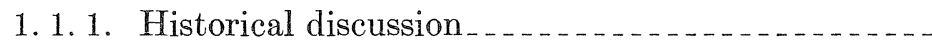

1. 2. Preparation of sugars $\ldots \ldots \ldots$

1.2. 1. D-Glucose $\ldots \ldots$

1. 2. 2. D-Fructose

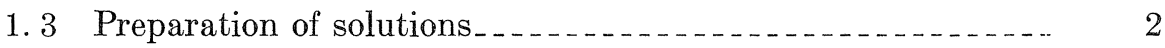

1.4. Determination of refractive indices ............... 2

1.4.1. Results .................................... 3

2. Density at various temperatures $\ldots$

2.1. Introduction $\ldots \ldots$

2. 1. 1. Historical discussion ..................... 4

2. 2. Determination of density 5

2.2.1. Results $\ldots \ldots$

3. References $\ldots \ldots \ldots$ 


\title{
Refractive Indices and Densities of Aqueous Solutions of Invert Sugar ${ }^{1}$
}

\author{
Carl F. Snyder and Albert T. Hattenburg
}

\begin{abstract}
The refractive indices and densities of aqueous solutions of invert sugar have been determined, at temperatures of $15,20,25$, and $30^{\circ} \mathrm{C}$, for concentrations up to about 82 percent of invert sugar (by weight). From the results, equations have been derived which relate the refractive index and percent of invert sugar (weight in air) at each temperature. Other equations relate the absolute density and percent of invert sugar (weight in vacuum) at each temperature. Five-decimal tables giving the refractive indices and densities of invert sugar solutions containing 1 to 85 percent of sugar are given for each percentage of invert sugar at the four temperatures.
\end{abstract}

\section{Refractive Index at Various Temperatures}

\section{Carl F. Snyder}

\subsection{Introduction}

The increasing use of refractometric methods for determining the dissolved solids in commercial, liquid sugar-products has made it desirable to obtain more-complete data on the refractive indices of aqueous solutions of pure invert sugar at various temperatures. These refractive indices have, therefore, been determined for concentrations up to about 82 percent (by weight), at 15, 20, 25, and $30^{\circ} \mathrm{C}$. From the results, an equation was derived relating refractive index and percent of invert sugar (by weight) at each temperature. From the four equations thus obtained, values of the index for equal increments of concentration were calculated.

\subsubsection{Historical Discussion}

Although fragmentary data on the refractive indices of solutions of some sugars had previously been reported, Stolle [1] ${ }^{2}$ is generally credited with the first systematic study of the refractive indices of sugars other than sucrose. He observed little difference in the indices of the different sugars in solutions of the same concentration. Tolman and Smith [2], using an Abbe refractometer, found that, for a number of sugars and related compounds, a solution of a given percentage by weight has approximately the same refractive index. In neither of these investigations were measurements made on solutions of invert sugar. Staněk and Vondrák [3] showed that the refractive index of an invert sugar solution is lower than that of a sucrose solution of the same concentration. The same observation was made by Schneller [4]. Most of the solutions measured by Schneller were prepared by the acid hydrolysis of sucrose and contained various proportions of invert sugar and

1 This Monograph is based on work partly supported by the Sugar Research Foundation, Inc.

2 Figures in brackets indicate the literature references at the end of this Monograph. sucrose. Subsequently, de Whalley [5] studied the refractometric estimation of dissolved solids in sirups containing invert sugar; he measured 17 solutions of invert sugar prepared by dissolving equal weights of pure D-glucose and D-fructose, for the range of 9.61 to 66.72 percent (by weight). The index was measured on an Abbe refractometer, and the observed refractive index was converted to percent solids by means of the table of the refractive indices of sucrose solutions. Both Schneller and de Whalley found that an appreciable difference exists between the refractive index of a solution of sucrose and one of invert sugar of the same concentration. Thus, estimation of the total solids in a sirup containing invert sugar (with ór without sucrose) by conversion of the observed refractive index to the equivalent sucrose (from the sucrose table) gave results which were too low. From his experimental results, de Whalley calculated an average correction of 0.022 for each percent of invert sugar present; this was to be added to the refractometric result for solids expressed as sucrose. Later, Zerban [6] showed that, for the data of both Schneller and de Whalley, the magnitude of the correction varies with the concentration. Zerban and Martin [7] then determined the refractive indices of invert sugar solutions at $20^{\circ} \mathrm{C}$ for the range of 0 to 80 percent (by weight), and devised the following equation where $p$ is the percent by weight of invert sugar solids.

$n_{D}{ }^{20}$ (invert sugar solutions)

$$
\begin{gathered}
=1.33299+1.411210 \times 10^{-3} p+0.5198919 \\
\times 10^{-5} p^{2}+1.56855 \times 10^{-8} p^{3} \\
-0.3776638 \times 10^{-10} p^{4} .
\end{gathered}
$$

Since the above measurements of the refractive index of invert sugar solutions were made only at $20{ }^{\circ} \mathrm{C}$, it was deemed desirable to repeat these measurements and to extend them to other temperatures. 


\subsection{Preparation of Sugars}

\subsubsection{D-Glucose}

The purest form of commercial dextrose hydrate that was readily available was dissolved in sufficient hot, distilled water to make a solution of approximately $60^{\circ} \mathrm{Brix}$; the solution was treated with veg'etable charcoal and filtered. The filtrate was warmed and refiltered through hardened filter-paper until all traces of charcoal had been removed. The final filtrate was concentrated under diminished pressure to a sirup containing: about 80 percent of dextrose (by weight); this was heated to $55{ }^{\circ} \mathrm{C}$, nucleated with anhydrous D-glucose, and placed in a rotating crystallizer enclosed in an insulated cabinet thermostated at $55^{\circ} \mathrm{C}$. The crystallization was usually complete within a few hours. The temperature within the cabinet was then lowered until the mass had reached a temperature of about 30 to $35^{\circ} \mathrm{C}$, and the crystals were separated from the mother liquor by centrifuging and thoroughly washed with absolute ethanol. Recrystallization was repeated until analysis of the product showed that the sugar was of the required purity. The final crystals were air-dried and then contained less than 0.1 percent of moisture. The sugar was finely powdered, dried at $60{ }^{\circ} \mathrm{C}$, and stored in vacuum desiccators over suitable drying agents.

\subsubsection{D-Fructose}

Relatively pure D-fructose (levulose) was available from the stock prepared at the National Bureau of Standards from tubers of the Jerusalem artichoke [8]. The sugar had already been recrystallized from water and it consisted of large crystals having a purity of over 99.9 percent. For this investigation, the sugar was further purified by the procedure of Jackson and Mathews [9]. A solution of the sugar in about an equal weight of water was treated with vegetable charcoal and the suspension was warmed and filtered. The filtrate was refiltered through hardened filterpaper to remove all traces of the charcoal, and concentrated at diminished pressure to a sirup containing 82 to 85 percent of 'D-fructose. The thick sirup was mixed with absolute ethanol and nucleated with crystals of D-fructose, and the suspension was rotated in a motor-driven apparatus at room temperature until crystallization was complete. The crystals were removed by centrifuging, thoroughly washed with absolute ethanol, air-dried, finely powdered, dried in a vacuum oven at $50{ }^{\circ} \mathrm{C}$ for about 1 hour, and stored in vacuum desiccators containing suitable desiccants.

\subsection{Preparation of Solutions}

The measurements of the refractive indices and densities were made on solutions prepared by dissolving a mixture of equal weights of pure D-glucose (dextrose) and pure D-fructose (levulose) in distilled water.
A suitable quantity of dry D-fructose was placed in a tared glass-stoppered flask, and the flask and contents were weighed and then dried to constant weight in a vacuum oven at $50{ }^{\circ} \mathrm{C}$. An equal weight of dry D-glucose was transferred to the flask, and the flask and contents were weighed and then dried in a vacuum oven at $50{ }^{\circ} \mathrm{C}$ to constant weight. The final weight of the contents represented the weight of dry invert sugar. Enough water to yield a solution of the desired concentration was added. The flask was mechanically rotated in an inclined position on an apparatus so constructed that its rotating shaft extended into an air bath fitted with heaters and a thermostat. For the solutions in the lower range of concentrations, elevated temperatures were not necessary, but, in the higher range, the air bath was heated to the lowest temperature that would ensure complete solution. When all of the sugar had dissolved, the flask was cooled to room temperature (if necessary) and weighed. From the weight of the solution and the weight of invert sugar taken, the composition of each solution was calculated in percent by weight (in air).

\subsection{Determination of Refractive Indices}

The solution of invert sugar was kept at room temperature for 24 hours to establish mutarotational equilibrium. Determinations of the refractive index were made with a Bausch and Lomb precision refractometer (Serial 6326), using a sodium lamp. For all readings, the instrument was adjusted to read (approximately) correctly for distilled water and the scale was calibrated by means of test plates of known refractive index. The instrument was kept in a room whose temperature was regulated to within about $1{ }^{\circ} \mathrm{C}$. Water from a large water-thermostat was continuously circulated through the prism jackets to maintain the same temperature of the solution and prisms to within 0.01 or $0.02{ }^{\circ} \mathrm{C}$.

For each series of measurements, the temperature of the room and water bath was adjusted to the desired value. A series of readings was taken with distilled water, the prism faces were thoroughly dried, and the instrument was filled with the solution of invert sugar. After a series of observations had been made on the solution, the prism faces were cleaned and a series of readings was taken with distilled water These operations were repeated until the required data had been obtained.

On completion of the series of measurements at one temperature, the temperature of the room and water bath was adjusted to a different temperature and a set of measurements was made at the new temperature. A random order was followed with respect to the measurements at different temperatures.

The observed readings on the instrument scale were converted to their equivalents in refractive index by means of the tables furnished with the instrument. 


\subsubsection{Results}

Measurements of the refractive indices were made on 39 solutions of invert sugar of concentrations ranging from about 5 to 82 percent by weight (in air). Each solution was measured at two or more of the selected temperatures (15, 20, 25, and $30^{\circ} \mathrm{C}$ ). From the data obtained at each temperature, an equation relating refractive index and percent of invert sugar by weight (in air) was derived (by the method of averages)

The four equations are as follows:

$$
\begin{aligned}
& n_{D}{ }^{15}=1.3333872+1.4292 \times 10^{-3} p \\
& \quad+0.515855 \times 10^{-5} p^{2}+0.1153 \times 10^{-7} p^{3} \\
& n_{D}{ }^{20}=1.3329877+1.4149 \times 10^{-3} p \\
& \quad+0.52729 \times 10^{-5} p^{2}+0.1105 \times 10^{-7} p^{3}
\end{aligned}
$$

$$
\begin{aligned}
& n_{D}{ }^{25}=1.3325026+1.4114 \times 10^{-3} p \\
&+ 0.51088 \times 10^{-5} p^{2}+0.1244 \times 10^{-7} p^{3} \\
& n_{D}{ }^{30}=1.3319403+1.4016 \times 10^{-3} p \\
&+0.5134 \times 10^{-5} p^{2}+0.1249 \times 10^{-7} p^{3}
\end{aligned}
$$

The experimental values obtained at each temperature for the invert sugar solutions of known composition and the values calculated from the corresponding equation are given in table 1.

\begin{tabular}{|c|c|c|c|c|c|c|c|c|c|c|c|c|}
\hline \multirow{2}{*}{$\begin{array}{l}\text { Invert sugar } \\
\text { by wt. (in } \\
\text { air) }\end{array}$} & \multicolumn{3}{|c|}{$n_{\mathrm{D}}$ at $15^{\circ} \mathrm{C}$} & \multicolumn{3}{|c|}{$n_{\mathrm{D}}$ at $20^{\circ} \mathrm{C}$} & \multicolumn{3}{|c|}{$n_{\mathrm{D}}$ at $25^{\circ} \mathrm{C}$} & \multicolumn{3}{|c|}{$n_{\mathrm{D}}$ at $30^{\circ} \mathrm{C}$} \\
\hline & Obs. & Calc. & Calc.-Obs. & Obs. & Calc. & Calc.-Obs. & Obs. & Calc. & Calc.-Obs. & Obs. & Calc. & Calc. $-O b$ \\
\hline $\begin{array}{r}\% \\
4.9106 \\
5.0244 \\
9.6687 \\
10.0548 \\
11.4836\end{array}$ & $\begin{array}{l}\text { 1. } 34049 \\
\text { 1. } 34076 \\
\text { 1. } 34768\end{array}$ & $\begin{array}{l}\text { 1. } 34053 \\
\text { 1. } 34070 \\
1.34770\end{array}$ & $\begin{array}{r}+0.00004 \\
+.00006 \\
+.00002\end{array}$ & $\begin{array}{l}\text { 1. } 34001 \\
\text { 1. } 34024 \\
1.34712 \\
1.34779 \\
1.34997\end{array}$ & $\begin{array}{l}1.34006 \\
1.34023 \\
1.34717 \\
1.34776 \\
1.34995\end{array}$ & $\begin{array}{r}+0.00005 \\
-.00001 \\
+.00005 \\
-.00003 \\
-.00002\end{array}$ & $\begin{array}{l}\text { 1. } 33956 \\
\text { 1. } 33973 \\
1.34665 \\
1.34724\end{array}$ & $\begin{array}{l}1.33956 \\
1.33972 \\
1.34664 \\
1.34722\end{array}$ & $\begin{array}{r}0.00000 \\
-.00001 \\
-.00001 \\
-.00002\end{array}$ & $\begin{array}{l}\text { 1. } 338888 \\
\text { 1. } 33913 \\
1.34593 \\
1.34871\end{array}$ & $\begin{array}{l}1,33895 \\
1.33911 \\
1.34598 \\
1.34873\end{array}$ & $\begin{array}{r}+0.00007 \\
-.00002 \\
+.00005 \\
+.00002\end{array}$ \\
\hline $\begin{array}{l}11.4961 \\
12.5735 \\
15.0320 \\
15.1660 \\
16.3219\end{array}$ & $\begin{array}{l}\text { 1. } 35055 \\
\text { 1. } 35225 \\
\text { 1. } 35615 \\
\text { 1. } 35627 \\
\text { 1. } 35805\end{array}$ & $\begin{array}{l}\text { 1. } 35052 \\
\text { 1. } 35220 \\
1.35608 \\
1.35629 \\
1.35814\end{array}$ & $\begin{array}{r}-.00003 \\
-.00005 \\
-.00007 \\
+.00002 \\
+.00009\end{array}$ & $\begin{array}{l}1.34994 \\
1.35171 \\
1.35542 \\
1.35570 \\
1.35753\end{array}$ & $\begin{array}{l}1.34997 \\
1.35163 \\
1.35549 \\
1.35570 \\
1.35754\end{array}$ & $\begin{array}{r}+.00003 \\
.00008 \\
+.00007 \\
.00000 \\
+.00001\end{array}$ & $\begin{array}{l}1.34936 \\
1.35109 \\
1.35499 \\
1.35504 \\
1.35698\end{array}$ & $\begin{array}{l}1.34942 \\
1.35108 \\
1.35492 \\
1.35513 \\
1.35696\end{array}$ & $\begin{array}{r}+.00006 \\
-.00001 \\
-.00007 \\
+.00009 \\
-.00002\end{array}$ & $\begin{array}{l}1.34871 \\
1.35051 \\
1.35420 \\
1.35433 \\
1.35629\end{array}$ & $\begin{array}{l}1.34875 \\
1.35040 \\
1.35421 \\
1.35442 \\
1.35624\end{array}$ & $\begin{array}{l}+.00004 \\
+.00011 \\
+.00001 \\
+.00009 \\
-.00005\end{array}$ \\
\hline $\begin{array}{l}16.5251 \\
18.7642 \\
20.0077 \\
20.0818 \\
25.0411\end{array}$ & $\begin{array}{l}\text { 1. } 35855 \\
\text { 1. } 36203 \\
1.36407 \\
1.37265\end{array}$ & $\begin{array}{l}1.35847 \\
1.36210 \\
1.36414 \\
1.37259\end{array}$ & $\begin{array}{l}-.00008 \\
+.00007 \\
+.00007 \\
-.00006\end{array}$ & $\begin{array}{l}1.35786 \\
1.36143 \\
1.36360 \\
1.36365 \\
1.37197\end{array}$ & $\begin{array}{l}1.35786 \\
1.36147 \\
1.36350 \\
1.36362 \\
1.37190\end{array}$ & $\begin{array}{r}.00000 \\
+.00004 \\
-.00010 \\
-.00003 \\
-.00007\end{array}$ & $\begin{array}{l}\text { 1. } 35729 \\
1.36085 \\
\text { 1. } 36290 \\
\text { 1. } 36305 \\
1.37128\end{array}$ & $\begin{array}{l}1.35728 \\
1.36087 \\
1.36289 \\
1.36301 \\
1.37125\end{array}$ & $\begin{array}{l}-.00001 \\
+.00002 \\
-.00001 \\
-.00004 \\
-.00003\end{array}$ & $\begin{array}{l}\text { 1. } 35656 \\
\text { 1. } 36010 \\
\text { 1. } 36228\end{array}$ & $\begin{array}{l}\text { 1. } 35656 \\
1.36013 \\
1.36214\end{array}$ & $\begin{array}{r}.00000 \\
+.00003 \\
-.00014\end{array}$ \\
\hline $\begin{array}{l}25.0691 \\
22.9445 \\
30.0336 \\
30.9705 \\
33.2872\end{array}$ & $\begin{array}{l}\text { 1. } 37268 \\
\text { 1. } 37581 \\
\text { 1. } 38138 \\
1.38283\end{array}$ & $\begin{array}{l}1.37264 \\
1.37587 \\
1.38128 \\
1.38294\end{array}$ & $\begin{array}{r}-.00004 \\
+.00006 \\
.00010 \\
+.00011\end{array}$ & $\begin{array}{l}1.37509 \\
\text { 1. } 38060 \\
1.38213 \\
1.38639\end{array}$ & $\begin{array}{l}1.37516 \\
1.38054 \\
1.38220 \\
1.38634\end{array}$ & $\begin{array}{l}+.00007 \\
+.00006 \\
+.00007 \\
-.00005\end{array}$ & $\begin{array}{l}\text { 1. } 37132 \\
\text { 1. } 37434 \\
\text { 1. } 37991 \\
\text { 1. } 38134 \\
1.38566\end{array}$ & $\begin{array}{l}1.37129 \\
1.37449 \\
1.37984 \\
1.38149 \\
1.38561\end{array}$ & $\begin{array}{r}-.00003 \\
+.00015 \\
-.00007 \\
+.00015 \\
-.00005\end{array}$ & $\begin{array}{l}1.37360 \\
1.38073\end{array}$ & $\begin{array}{l}1.37368 \\
1.38064\end{array}$ & $\begin{array}{l}+.00008 \\
-.00009\end{array}$ \\
\hline $\begin{array}{l}34.9011 \\
39.3212 \\
42.2839 \\
45.0871 \\
50.1960\end{array}$ & $\begin{array}{l}1.38994 \\
1.40390 \\
\text { 1. } 40946 \\
1.41958\end{array}$ & $\begin{array}{l}1.39004 \\
1.40391 \\
\text { 1. } 40937 \\
1.41958\end{array}$ & $\begin{array}{r}+.00010 \\
+.00001 \\
-.00009 \\
.00000\end{array}$ & $\begin{array}{l}1.38929 \\
1.39753 \\
1.40299 \\
1.41869\end{array}$ & $\begin{array}{l}1.38926 \\
1.39745 \\
1.40308 \\
1.41870\end{array}$ & $\begin{array}{l}-.00003 \\
-.00008 \\
+.00009 \\
+.00001\end{array}$ & $\begin{array}{l}1.38864 \\
1.39678 \\
1.40223 \\
1.41774\end{array}$ & $\begin{array}{l}1.38852 \\
1.39666 \\
1.40226 \\
1.41780\end{array}$ & $\begin{array}{l}-.00012 \\
-.00012 \\
+.00003 \\
+.00006\end{array}$ & $\begin{array}{l}1.38761 \\
1.40124 \\
1.41680\end{array}$ & $\begin{array}{l}1.38764 \\
1.40133 \\
1.41681\end{array}$ & $\begin{array}{l}+.00003 \\
+.00009 \\
+.00001\end{array}$ \\
\hline $\begin{array}{l}52.4589 \\
55.2430 \\
58.8565 \\
60.3033 \\
64.9237\end{array}$ & $\begin{array}{l}\text { 1. } 42420 \\
\text { 1. } 43005 \\
\text { 1. } 43771 \\
\text { 1. } 44090 \\
1.45114\end{array}$ & $\begin{array}{l}1.42422 \\
1.43003 \\
\text { 1. } 43773 \\
1.44086 \\
1.45108\end{array}$ & $\begin{array}{l}+.00002 \\
-.00002 \\
+.00002 \\
-.00004 \\
-.00006\end{array}$ & $\begin{array}{l}1.42325 \\
1.42909 \\
1.43677 \\
1.43994 \\
1.45017\end{array}$ & $\begin{array}{l}\text { 1. } 42332 \\
\text { 1. } 42911 \\
\text { 1. } 43679 \\
\text { 1. } 43991 \\
1.45010\end{array}$ & $\begin{array}{l}+.00007 \\
+.00002 \\
+.00002 \\
-.00003 \\
-.00007\end{array}$ & $\begin{array}{l}1.42230 \\
1.42810 \\
1.43583 \\
1.43900 \\
1.44923\end{array}$ & $\begin{array}{l}1.42240 \\
1.42816 \\
1.43581 \\
1.43892 \\
1.44908\end{array}$ & $\begin{array}{l}+.00010 \\
+.00006 \\
-.00002 \\
-.00008 \\
-.00015\end{array}$ & $\begin{array}{l}1.42134 \\
1.42709 \\
1.43484 \\
1.43804 \\
1.44814\end{array}$ & $\begin{array}{l}1.42140 \\
1.42714 \\
1.43477 \\
1.43787 \\
1.44800\end{array}$ & $\begin{array}{l}+.00006 \\
+.00005 \\
-.00007 \\
-.00017 \\
-.00014\end{array}$ \\
\hline $\begin{array}{l}65.1626 \\
68.6833 \\
70.7986 \\
73.1294 \\
75.5289\end{array}$ & $\begin{array}{l}\text { 1. } 45166 \\
\text { 1. } 45974 \\
\text { 1. } 46454 \\
\text { 1. } 47010 \\
1.47563\end{array}$ & $\begin{array}{l}\text { 1. } 45161 \\
\text { 1. } 45962 \\
1.46452 \\
1.47000 \\
1.47573\end{array}$ & $\begin{array}{l}-.00005 \\
-.00012 \\
-.00002 \\
-.00010 \\
+.00010\end{array}$ & $\begin{array}{l}1.45070 \\
1.45880 \\
1.46359 \\
1.46898 \\
1.47482\end{array}$ & $\begin{array}{l}\text { 1. } 45064 \\
\text { 1. } 45863 \\
1.46352 \\
1.46899 \\
1.47470\end{array}$ & $\begin{array}{l}-.00006 \\
-.00017 \\
-.00007 \\
+.00001 \\
-.00012\end{array}$ & $\begin{array}{l}\text { 1. } 45772 \\
\text { 1. } 46249 \\
1.46786 \\
1.46365\end{array}$ & $\begin{array}{l}1.45758 \\
1.46245 \\
1.46791 \\
1.46361\end{array}$ & $\begin{array}{r}-.00014 \\
-.00004 \\
+.00005 \\
-.00004\end{array}$ & $\begin{array}{l}1.44859 \\
1.45656 \\
1.46137 \\
1.46685\end{array}$ & $\begin{array}{l}1.44853 \\
1.45648 \\
1.46134 \\
1.46678\end{array}$ & $\begin{array}{l}-.00006 \\
-.00008 \\
-.00003 \\
-.00007\end{array}$ \\
\hline $\begin{array}{l}75.5960 \\
78.1373 \\
80.1473 \\
82.7504\end{array}$ & $\begin{array}{l}\text { 1. } 47584 \\
\text { 1. } 48203 \\
\text { 1. } 48687 \\
\text { 1. } 49347\end{array}$ & $\begin{array}{l}\text { 1. } 47589 \\
\text { 1. } 48206 \\
\text {. } 48701 \\
1.49351\end{array}$ & $\begin{array}{r}+.00005 \\
+.00003 \\
+.00014 \\
+.00004\end{array}$ & $\begin{array}{l}1.47494 \\
1.48096 \\
1.48568 \\
1.49221\end{array}$ & $\begin{array}{l}\text { 1. } 47486 \\
\text { 1. } 48102 \\
\text { 1. } 48596 \\
1.49245\end{array}$ & $\begin{array}{r}-.00008 \\
+.00006 \\
+.00028 \\
+.00024\end{array}$ & $\begin{array}{l}\text { 1. } 47385 \\
\text { 1. } 47984 \\
1.48469 \\
1.49114\end{array}$ & $\begin{array}{l}1.47377 \\
1.47992 \\
1.48485 \\
1.49133\end{array}$ & $\begin{array}{r}-.00008 \\
+.00008 \\
+.00016 \\
+.00019\end{array}$ & $\begin{array}{l}1.47268 \\
1.47867 \\
1.48365 \\
1.48987\end{array}$ & $\begin{array}{l}\text { 1. } 47263 \\
1.47877 \\
1.48369 \\
1.49016\end{array}$ & $\begin{array}{r}-.00005 \\
+.00010 \\
+.00004 \\
+.00029\end{array}$ \\
\hline
\end{tabular}

By employing equations (2) to (5), the refractive indices at equal increments of concentration were calculated; they are given in table 2 . These values (table 2) for the refractive indices at $20{ }^{\circ} \mathrm{C}$ are slightly higher than those reported by Zerban and Martin [7].

TABLE 1. Refractive indices of invert sugar solutions 
TABLE 2. Refractive indices of inveri sugar solutions*

\begin{tabular}{|c|c|c|c|c|}
\hline \multirow{2}{*}{$\begin{array}{l}\text { Invert } \\
\text { sugar by } \\
\text { weight } \\
\text { (in air) }\end{array}$} & \multicolumn{4}{|c|}{$n_{\mathrm{D}}$ at } \\
\hline & $15^{\circ} \mathrm{C}$ & $20^{\circ} \mathrm{C}$ & $25^{\circ} \mathrm{C}$ & $30^{\circ} \mathrm{C}$ \\
\hline$\%$ & & & & \\
\hline 0 & 1. 33339 & 1. 33299 & 1. 33250 & 1. 33194 \\
\hline $\begin{array}{l}1 \\
2\end{array}$ & $\begin{array}{l}1.33482 \\
133627\end{array}$ & $\begin{array}{l}\text { 1. } 33441 \\
1.33584\end{array}$ & $\begin{array}{l}1.33392 \\
1.33535\end{array}$ & $\begin{array}{l}\text { 1. } 33335 \\
1.33476\end{array}$ \\
\hline$\frac{2}{3}$ & 1. 33772 & 1. 33728 & 1. 33678 & 1. 33619 \\
\hline 4 & 1. 33919 & 1. 33873 & 1. 33823 & 1. 33763 \\
\hline 5 & 1. 34066 & 1. 34020 & 1.33969 & 1. 33908 \\
\hline 6 & 1. 34215 & 1.34167 & 1. 34116 & 1. 34054 \\
\hline 7 & 1. 34364 & 1. 34315 & 1. 34264 & 1. 34201 \\
\hline 8 & 1. 34516 & 1. 34465 & 1. 34413 & 1. 34349 \\
\hline 9 & 1. 34668 & 1. 34616 & 1. 34563 & 1. 34498 \\
\hline 10 & 1. 34821 & 1. 34768 & 1. 34714 & 1. 34648 \\
\hline 11 & 1. 34975 & 1. 34920 & 1.34866 & 1. 34800 \\
\hline 12 & 1. 35130 & 1. 35075 & 1. 35020 & 1. 34952 \\
\hline 13 & 1. 35286 & 1. 35230 & 1. 35174 & 1. 35106 \\
\hline 14 & 1. 35444 & 1. 35386 & 1. 35330 & 1. 35260 \\
\hline 15 & 1. 35603 & 1. 35544 & 1. 35487 & 1. 35416 \\
\hline 16 & 1. 35762 & 1. 35702 & 1. 35644 & 1. 35573 \\
\hline 17 & 1. 35923 & 1. 35862 & 1. 35803 & 1. 35731 \\
\hline 18 & 1 . 2Rnes & 1. 36023 & 1. 35964 & 1. 35891 \\
\hline 19 & 1. 36248 & 1. 36185 & 1. 36125 & 1. 36051 \\
\hline 20 & 1. 36413 & 1. 36348 & 1. 36287 & 1. 36213 \\
\hline 21 & 1. 36578 & 1. 36513 & 1. 36451 & 1. 36375 \\
\hline 22 & 1. 36745 & 1. 36679 & 1. 36616 & 1. 36539 \\
\hline 23 & 1. 36913 & 1. 36846 & 1. 36782 & 1. 36705 \\
\hline 24 & 1. 37082 & 1. 37014 & 1. 36949 & 1. 36871 \\
\hline 25 & 1. 37252 & 1. 37183 & 1. 37118 & 1.37038 \\
\hline 26 & 1. 37424 & 1. 37354 & 1. 37287 & 1. 37207 \\
\hline 27 & 1. 37596 & 1. 37525 & 1. 37458 & 1. 37377 \\
\hline 28 & 1. 37770 & 1. 37698 & 1. 37630 & 1. 37548 \\
\hline 29 & 1. 37945 & 1.37873 & 1. 37803 & 1. 37721 \\
\hline 30 & 1. 381 & 1. 38048 & 1. 37978 & 1.37895 \\
\hline 31 & 1. 38299 & 1. 38225 & 1. 38154 & 1. 38070 \\
\hline 32 & 1. 38478 & 1. 38403 & 1. 38331 & 1.38246 \\
\hline 33 & 1. 38658 & 1. 38582 & 1. 38509 & 1. 38423 \\
\hline 34 & 1. 38840 & 1. 38763 & 1. 38689 & 1. 38602 \\
\hline 35 & 1. 39022 & 1. 38944 & 1. 38869 & 1. 38782 \\
\hline 36 & 1. 39206 & 1. 39128 & 1. 39052 & 1. 38964 \\
\hline 37 & 1. 39391 & 1. 39312 & 1. 39235 & 1. 39146 \\
\hline 38 & 1. 39578 & 1. 39498 & 1. 39420 & 1. 39330 \\
\hline & 1. 39766 & 1.39685 & 1. 39606 & 1. 39515 \\
\hline 40 & 1.3 & 1. & 1. & 1. 39702 \\
\hline 41 & 1. 40145 & 1.40063 & 1. 39982 & 1. 39890 \\
\hline 42 & 1. 40337 & 1. 40254 & 1. 40172 & 1. 40079 \\
\hline 43 & 1. 40530 & 1. 40446 & 1. 40363 & 1. 4027 \\
\hline 44 & 1.40724 & 1. 40640 & 1. 40556 & 1. 40462 \\
\hline
\end{tabular}

TABLE 2--Continued

\begin{tabular}{|c|c|c|c|c|}
\hline \multirow{2}{*}{$\begin{array}{l}\text { Invert } \\
\text { sugar by } \\
\text { weight } \\
\text { (in air) }\end{array}$} & \multicolumn{4}{|c|}{$n_{\mathrm{D}}$ at } \\
\hline & $15^{\circ} \mathrm{C}$ & $20^{\circ} \mathrm{C}$ & $25^{\circ} \mathrm{C}$ & $30^{\circ} \mathrm{C}$ \\
\hline 45 & 1. 40920 & 1. 40835 & 1.40750 & 1. 40655 \\
\hline 46 & 1. 41117 & 1. 41031 & 1. 40945 & 1. 40849 \\
\hline 47 & 1. 41315 & 1. 41229 & 1. 41142 & 1. 41045 \\
\hline 48 & 1.41515 & 1. 41428 & 1. 41340 & 1. 41243 \\
\hline 49 & 1. 41716 & 1. 41628 & 1. 41539 & 1.41442 \\
\hline 50 & 1. 41919 & 1. 41830 & 1. 41740 & 1. 41642 \\
\hline 51 & 1. 42122 & 1. 42033 & 1. 41942 & 1. 41843 \\
\hline 52 & 1. 42328 & 1. 42238 & 1. 42146 & $\begin{array}{l}1.42046 \\
1.0\end{array}$ \\
\hline 53 & 1. 42534 & 1. 42444 & 1. 42351 & 1.42251 \\
\hline 54 & 1. 42742 & 1. 42651 & 1. 42558 & 1.42457 \\
\hline 55 & 1.42952 & 1. 42860 & 1. 42766 & 1.42664 \\
\hline 56 & 1. 43163 & 1. 43070 & 1. 42975 & 1.42873 \\
\hline 57 & 1. 43375 & 1. 43282 & 1. 43186 & 1.43083 \\
\hline 58 & 1.43589 & 1. 43495 & 1. 43398 & 1. 43294 \\
\hline 59 & 1. 43804 & 1. 43710 & 1. 43612 & 1.43507 \\
\hline 60 & 1.44020 & 1. 43926 & 1. 43827 & 1. 43722 \\
\hline 61 & 1. 44238 & 1. 44143 & 1. 44043 & 1. 43938 \\
\hline 62 & 1. 44458 & 1. 44362 & 1. 44262 & 1.44155 \\
\hline 63 & 1. 44679 & 1. 44582 & 1. 44481 & 1.44374 \\
\hline 64 & 1. 44901 & 1. 44804 & 1. 44702 & $\begin{array}{l}1.44595 \\
.\end{array}$ \\
\hline 65 & 1.45125 & 1. 45027 & 1. 44925 & 1.44817 \\
\hline 66 & 1. 45350 & 1. 45252 & 1. 45149 & 1.45040 \\
\hline 67 & 1.45577 & 1.45479 & 1. 45375 & 1. 45265 \\
\hline 68 & 1. 45805 & 1. 45706 & 1. 45602 & 1. 45492 \\
\hline 69 & 1. 46035 & 1. 45936 & 1. 45830 & 1.45720 \\
\hline 70 & 1. 46266 & 1. 46166 & 1. 46060 & 1.45950 \\
\hline 71 & 1. 46499 & 1. 46399 & 1. 46292 & 1. 46181 \\
\hline 72 & 1. 46734 & 1. 46633 & 1. 46525 & 1.46414 \\
\hline 73 & 1. 46970 & 1. 46868 & 1. 46760 & 1.46648 \\
\hline 74 & 1.47207 & 1. 47105 & 1. 46997 & 1. 46884 \\
\hline 75 & 1. 47446 & 1. 47343 & 1. 47235 & $\begin{array}{l}1.47121 \\
\text {. }\end{array}$ \\
\hline 76 & 1.47687 & 1. 47583 & 1. 47474 & 1. 47360 \\
\hline 77 & 1. 47929 & 1. 47825 & 1. 47715 & 1. 47601 \\
\hline 78 & 1.48172 & 1. 48068 & 1.47958 & 1.47843 \\
\hline 79 & 1.48418 & 1.48313 & 1. 48203 & 1. 48087 \\
\hline 80 & 1. 48664 & 1. 48559 & 1.48449 & 1.48333 \\
\hline 81 & 1.48913 & 1. 48807 & 1. 48696 & 1. 48580 \\
\hline 82 & 1.49163 & 1. 49057 & 1. 48945 & 1.48828 \\
\hline 83 & 1. 49414 & 1. 49308 & 1. 49196 & $\begin{array}{l}1.49079 \\
\end{array}$ \\
\hline 84 & 1. 49668 & 1.49560 & 1. 49449 & 1. 49331 \\
\hline 85 & 1.49922 & 1. 49815 & 1.49703 & 1. 49584 \\
\hline
\end{tabular}

*A condensed form of this table was published in the Proceedings of the Twelfth Session of the International Commission for Uniform Methods of Sugar Analysis, 1958, page 26.

\section{Density at Various Temperatures}

\section{Albert T. Hattenburg and Carl F. Snyder}

\subsection{Introduction}

Densimetric methods are applicable to the determination of dissolved solids in commercial liquid-sugar products. The true densities of 15 solutions of invert sugar of various concentrations were determined at $15,20,25$, and $30{ }^{\circ} \mathrm{C}$. By using the experimental data at each of these temperatures, an equation was derived relating true density and percent of invert sugar by weight (in vacuum). From the four equations thus obtained, the values of the density at equal increments of concentration (percent of invert sugar by weight) were calculated.

\subsubsection{Historical Discussion}

The determination of the densities of sugar solutions of various concentrations and temperatures has been the subject of extensive investigation. Because of the commercial importance of sucrose, the densities of its aqueous solutions have received the most attention. Since their publication in 1900, Plato, Domke, and Harting's tables of densities of sucrose solutions [10] have been generally accepted as being the most accurate that were available. Although, strictly, these tables are applicable to solutions of pure sucrose only, they are frequently employed for expressing the total content of dissolved solids in a sugar solution containing other dissolved solids besides sucrose. For such materials, the values have been expressed as apparent percent of sucrose or as "sucrose equivalent."

By basing calculations on the values of Plato and co-workers [10] for the densities of sucrose solutions, many other tables have been prepared. Among these is the expanded table of Sidersky [11] which gives the number of grams of sucrose per $100 \mathrm{~g}$ and per $100 \mathrm{ml}$ of solution for concentrations up to 30 percent by weight at $\mathrm{t} / 4{ }^{\circ} \mathrm{C}$ and t/15 ${ }^{\circ} \mathrm{C}$ (between 10 and $30^{\circ}$ ). Domke [12] published a table of apparent specific gravities at 
$20^{\circ} \mathrm{C} / 20{ }^{\circ} \mathrm{C}$ for aqueous solutions containing 0 to 90 percent of sucrose by weight. Bates and Bearce [13] calculated a table giving the Baume equivalents in specific gravities at $20{ }^{\circ} \mathrm{C} / 4{ }^{\circ} \mathrm{O}$ and $20^{\circ} \mathrm{C} / 20^{\circ} \mathrm{C}$. A table of weights of sucrose per gallon and per cubic foot of aqueous solutions was computed by Snyder and Hammond [14].

For use in the tropics, a table of specific gravities of sucrose solutions at $27.5{ }^{\circ} \mathrm{C} / 4{ }^{\circ} \mathrm{C}$ was calculated by Dowes-Dekkar and Erlee [15] and at $28{ }^{\circ} \mathrm{C} / 4^{\circ} \mathrm{C}$ by Sidersky [11]. For use in Hawaii, a table giving apparent specific gravities at 27.5 ${ }^{\circ} \mathrm{C} / 27.5{ }^{\circ} \mathrm{C}$ was given in the Methods of Chemical Control of the Association of Hawaiian Sugar Technologists.

The densities of aqueous solutions containing up to 30 percent of D-glucose were determined by Jackson [16] and by Riiber [17]. Subsequently, Jackson and Matthews [9] determined the densities of aqueous solutions containing up to 70 percent of $\mathrm{D}$-fructose.

The lack of published data on the densities of aqueous solutions of invert sugar led to the experimental determinations now reported.

\subsection{Determination of Density}

Data on the densities of invert sugar solutions were obtained from a series of determinations made at $15,20,25$, and $30{ }^{\circ} \mathrm{C}$, for solutions of 15 different concentrations prepared as described in section 1.3. The weight of invert sugar and the weight of the solution (in air) of each of the solutions were converted to weights in vacuum, and the percent (by weight) of invert sugar (in vacuum) was calculated. The measurements were made by the use of a pycnometer which consisted of a long cylindrical gluss body of about 100-inl capacity. Access to the body was provided by two capillary tubes whose upper ends protruded into a small bowl fitted with a ground-glass cover. The small bowl served to contain the solution which spilled out of the tubes upon removal from a high-temperature bath.

\subsubsection{Results}

From the data at each temperature, an equation relating absolute density (in $\mathrm{g} / \mathrm{ml}$ ) and percent of invert sugar by weight (in vacuum) was derived by the method of averages.

The four equations are as follows:

$$
\begin{aligned}
& \mathrm{d}^{15^{\circ}}=0.9991266+0.3895362 \times 10^{-2} q+0.124621 \\
& \times 10^{-4} q^{2}+0.4632 \times 10^{-7} q^{3}-0.21854 \times 10^{-9} q^{4}
\end{aligned}
$$

$\mathrm{d}^{20^{\circ}}=0.9982343+0.3863641 \times 10^{-2} q+0.1214466$

$\times 10^{-4} q^{2}+0.5417 \times 10^{-7} q^{3}-0.25882 \times 10^{-9} q^{4}$

$\mathrm{d}^{25^{\circ}}=0.9970770+0.3827780 \times 10^{-2} q+0.1261903$

$\times 10^{-4} q^{2}+0.4235 \times 10^{-7} q^{3}-0.1661 \times 10^{-9} q^{4}$

$\mathrm{d}^{30^{\circ}}=0.9956780+0.3798374 \times 10^{-2} q+0.1264516$

$\times 10^{-4} q^{2}+0.4180 \times 10^{-7} q^{3}-0.1533 \times 10^{-9} q^{4}$

Table 3 shows the observed densities for each concentration at each temperature and the values calculated from the corresponding equation.

By means of equations (6) to (9), the absolute densities at equal increments of concentration were calculated; they are given in table 4.

\begin{tabular}{|c|c|c|c|c|c|c|c|c|c|c|c|c|}
\hline \multirow{3}{*}{$\begin{array}{l}\text { Invert } \\
\text { sugar by } \\
\text { wt. (in } \\
\text { vacuo) }\end{array}$} & \multicolumn{12}{|c|}{ Density $(\mathrm{g} / \mathrm{ml})$ at } \\
\hline & \multicolumn{3}{|c|}{$15^{\circ} \mathrm{C}$} & \multicolumn{3}{|c|}{$20^{\circ} \mathrm{C}$} & \multicolumn{3}{|c|}{$25^{\circ} \mathrm{C}$} & \multicolumn{3}{|c|}{$30^{\circ} \mathrm{C}$} \\
\hline & Obs. & Calc. & Calc.-Obs. & Obs. & Calc. & Calc.-Obs. & Obs. & Calc. & Calc.-Obs. & Obs. & Calc. & Calc.-Obs. \\
\hline $\begin{array}{r}\% \\
4.9086 \\
9.6649 \\
11.4917 \\
15.1604 \\
18.7575\end{array}$ & $\begin{array}{l}1.01857 \\
1.03798 \\
1.04563 \\
1.06116 \\
1.07685\end{array}$ & $\begin{array}{l}1.01855 \\
1.03798 \\
1.04560 \\
1.06120 \\
1.07686\end{array}$ & $\begin{array}{r}-0.00002 \\
.00000 \\
-.00003 \\
+.00004 \\
.+.00001\end{array}$ & $\begin{array}{l}\text { 1. } 01754 \\
\text { 1. } 03674 \\
1.04436 \\
1.05972 \\
1.07532\end{array}$ & $\begin{array}{l}\text { 1. } 01750 \\
1.03676 \\
1.04432 \\
1.05978 \\
1.07531\end{array}$ & $\begin{array}{r}-0.00004 \\
+.00002 \\
+.00004 \\
+.00006 \\
-.00001\end{array}$ & $\begin{array}{l}\text { 1. } 01619 \\
1.03527 \\
1.04282 \\
1.05812 \\
1.07358\end{array}$ & $\begin{array}{l}\text { 1. } 01617 \\
1.03529 \\
1.04279 \\
1.05815 \\
1.07358\end{array}$ & $\begin{array}{r}-0.00002 \\
+.00002 \\
+.00003 \\
+.00003 \\
.00000\end{array}$ & $\begin{array}{l}1.01462 \\
1.03360 \\
1.04110 \\
1.05629 \\
1.07165\end{array}$ & $\begin{array}{l}1.01463 \\
1.03361 \\
1.04106 \\
1.05631 \\
1.07163\end{array}$ & $\begin{array}{r}+0.00001 \\
+.00001 \\
+.00004 \\
+.00002 \\
-.00002\end{array}$ \\
\hline $\begin{array}{l}26.9360 \\
30.9614 \\
34.8913 \\
42.2733 \\
52.4482\end{array}$ & $\begin{array}{l}1.11394 \\
1.13282 \\
1.15184 \\
1.18875 \\
1.24276\end{array}$ & $\begin{array}{l}1.11388 \\
1.13285 \\
1.15186 \\
1.18887 \\
1.24274\end{array}$ & $\begin{array}{r}-.00006 \\
+.00003 \\
+.00002 \\
+.00012 \\
-.00002\end{array}$ & $\begin{array}{l}1.11204 \\
1.13085 \\
1.14975 \\
1.18653 \\
1.24023\end{array}$ & $\begin{array}{l}1.11204 \\
1.13087 \\
1.14974 \\
1.18653 \\
1.24014\end{array}$ & $\begin{array}{r}.00000 \\
+.00002 \\
-.00001 \\
.00000 \\
-.00009\end{array}$ & $\begin{array}{l}\text { 1. } 11010 \\
1.12875 \\
1.14757 \\
1.18408 \\
1.23743\end{array}$ & $\begin{array}{l}1.11008 \\
1.12879 \\
1.14755 \\
1.18411 \\
1.23740\end{array}$ & $\begin{array}{r}r .00002 \\
+.00004 \\
+.00002 \\
+.00003 \\
-.00033\end{array}$ & $\begin{array}{l}1.10790 \\
1.12650 \\
1.14514 \\
1.18150 \\
1.23458\end{array}$ & $\begin{array}{l}1.10790 \\
1.12650 \\
1.14515 \\
1.18151 \\
1.23455\end{array}$ & $\begin{array}{r}.00000 \\
.00000 \\
+.00001 \\
+.00001 \\
-.00003\end{array}$ \\
\hline $\begin{array}{l}58.8461 \\
64.9138 \\
70.7899 \\
75.5211 \\
82.7439\end{array}$ & $\begin{array}{l}1.27846 \\
1.31327 \\
1.34828 \\
1.37725 \\
1.42273\end{array}$ & $\begin{array}{l}1.27833 \\
1.31329 \\
1.34827 \\
1.37723 \\
1.42276\end{array}$ & $\begin{array}{r}-.00013 \\
+.00002 \\
-.00001 \\
-.00002 \\
+.00003\end{array}$ & $\begin{array}{l}\text { 1. } 27564 \\
1.31030 \\
1.34531 \\
1.37418 \\
1.41967\end{array}$ & $\begin{array}{l}\text { 1. } 27559 \\
\text { 1. } 31044 \\
1.34532 \\
1.37420 \\
1.41964\end{array}$ & $\begin{array}{r}-.00005 \\
+.00014 \\
+.00001 \\
+.00002 \\
-.00003\end{array}$ & $\begin{array}{l}1.27276 \\
1.30728 \\
1.34216 \\
1.37094 \\
1.41642\end{array}$ & $\begin{array}{l}\text { 1. } 27266 \\
\text { 1. } 30736 \\
1.34214 \\
1.37097 \\
1.41641\end{array}$ & $\begin{array}{r}-.00010 \\
+.00008 \\
+.00002 \\
+.00003 \\
-.00001\end{array}$ & $\begin{array}{l}\text { 1. } 26971 \\
1.30418 \\
1.33890 \\
1.36764 \\
1.41309\end{array}$ & $\begin{array}{l}1.26967 \\
1.30424 \\
1.33891 \\
1.36768 \\
1.41304\end{array}$ & $\begin{array}{r}-.00004 \\
+.00006 \\
+.00001 \\
+.00004 \\
-.00005\end{array}$ \\
\hline
\end{tabular}

TABLE 3. Densities of invert sugar solutions 
TABLE 4. Densities of invert sugar solutions

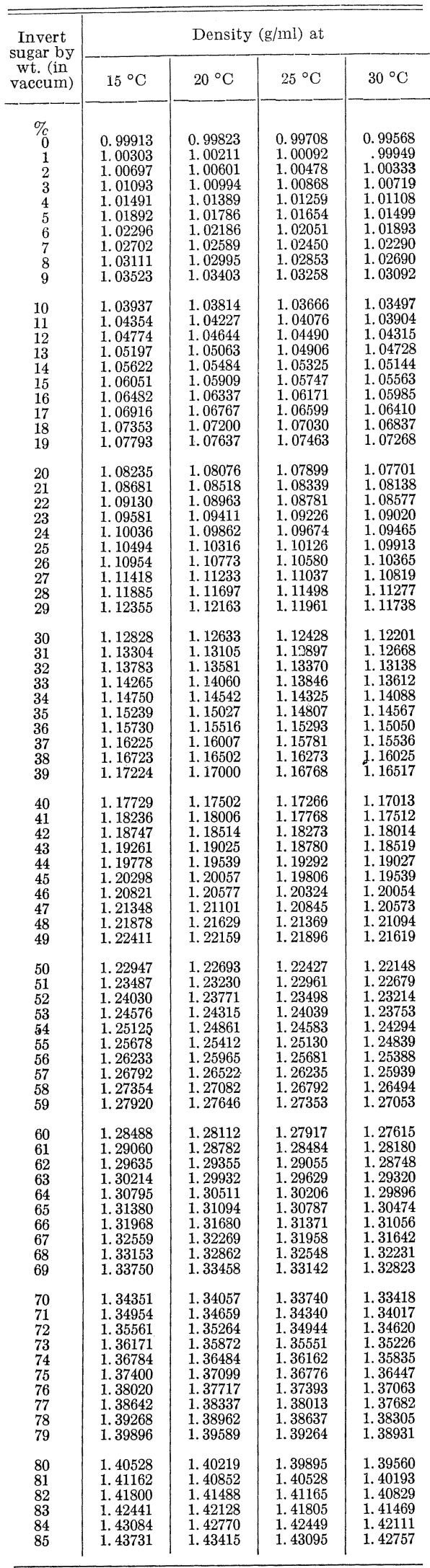

The authors gratefully acknowledge the help of Charles $\mathrm{T}$ Collett who designed the pycnometer used for the measurements of density and who rendered advice and assistance while the work was in progress.

\section{References}

[1] F. Stolle, Z. Ver. deut. Zücker-Ind. 51, 469 (1901).

[2] L. M. Tolman and W. B. Smith, J. Am. Chem. Soc. 28, 1476 (1906).

[3] V. Staněk and J. Vondrák, Z. Zuckerind. čechoslov. Rep. 45, 203 (1920-1921).

[4] M. Schneller, J. Assoc. Offic. Agr. Chemists 9, 156 (1926).

[5] H. C. S. deWhalley, Intern Sugar J. 37, 353 (1935).

[6] F. W. Zerban, J. Assn. Official Agr. Chem. 26, 143 (1943).

[7] F. W. Zerban and J. Martin, J. Assoc. Offic. Agr. Chemists 2\%, 295 (1944)

[8] R. F. Jackson, C. G. Silsbee, and M. J. Proffitt, B. S. Sci. Paper 519 (1926).

[9] R. F. Jackson and J. A. Mathews, BS J. Research 8, 404 (1932) RP426.

[10] F. Plato, J. Domke, and H. Harting, Z. Ver. deut. Zücker-Ind. 50, 982, 1079 (1900).

111] D. Sidersky, Les densités des solutions sucrées à différentes températures, Paris (1908).

[12] J. Domke, Z. Ver. deut. Zücker-Ind. 62, 302 (1912).

[13] F. J. Bates and H. W. Bearce, BS Tech. Paper, T115 (1918).

[14] C. F. Snyder and L. D. Hammond, NBS Circular C45y (1946)

[15] K. Dowes-Dekker and T. J. D. Erlee, Arch. Suikerind. Ned. en Ned.-Indië 38, III 697 (1930).

[16] R. F. Jackson, BS Sci. Paper 293 (1916).

[17] C. N. Riiber, Ber. 56, 2185 (1923). 


\section{U.S. DEPARTMENT OF COMMERCE}

Luther H. Hodges, Secretary

NATIONAL BUREAU OF STANDARDS

A. V. Astin, Director

\section{THE NATIONAL BUREAU OF STANDARDS}

The scope of activities of the National Bureau of Standards at its major laboratories in Washington, D.C., and Boulder, Colorade, is suggested in the following listing of the divisions and sections engaged in technical work. In general, each section carries out specialized research, development, and engineering in the field indicated by its title. A brief description of the activities, and of the resultant publications, appears on the inside of the front cover.

\section{WASHINGTON, D.C.}

Electricity. Resistance and Reactance. Electrochemistry. Electrical Instruments. Magnetic Measurements. Dielectrics. High Voltage. Absolute Electrical Measurements.

Metrology. Photometry and Colorimetry. Refractometry. Photographic Research. Length. Engineering Metrology. Mass and Scale. Volumetry and Densimetry.

Heat. Temperature Physics. Heat Measurements. Cryogenic Physics. Equation of State. Statistical Physics.

Radiation Physics. X-ray. Radioactivity. Radiation Theory. High Energy Radiation. Radiological Equipment. Nucleonic Instrumentation. Neutron Physics.

Analytical and Inorganic Chemistry. Pure. Substances. Spectrochemistry. Solution Chemistry. Standard Reference Materials. Applied Analytical Research. Crystal Chemistry.

Mechanics. Sound. Pressure and Vacuum. Fluid Mechanics. Engineering Mechanics. Rheology. Combustion Controls.

Polymers. Macromolecules: Synthesis and Structure. Polymer Chemistry. Polvmer Physics. Polymer Characterization. Polvmer Evaluation and Testing. Applied Polymer Standards and Research. Dental Research. Metallurgy. Engineering Metallurgy. Microscopy and Diffraction. Metal Reactions. Metal Physics. Electrolysis and Metal Deposition.

Inorganic Solids. Engineering Ceramics. Glass. Solid State Chemistry. Crystal Growth. Physical Properties. Crystallography.

Building Research. Structural Engineering. Fire Research. Mechanical Systems. Organic Building Materials. Codes and Safety Standards. Heat Transfer. Inorganic Building Materials. Metallic Building Materials.

Applied Mathematics. Numerical Analysis. Computation. Statistical Engineering. Mathematical Physics. Operations Research.

Data Processing Systems. Components and Techniques. Computer Technology. Measurements Automation. Engineering Applications. Systems Analysis.

Atomic Physics. Spectroscopy. Infrared Spectroscopy. Solid State Physics. Electron - Physics. Atomic Physics.

Instrumentation. Engineering Electronics. Electron Devices. Electronic Instrumentation. Mechanical Instruments. Basic Instrumentation.

Physical Chemistry. Thermochemistry. Surface Chemistry. Organic Chemistry. Molecular Spectroscopy. Elementary Processes. Mass Spectrometry. Photochemistry and Radiation Chemistry.

Office of Weights and Measures.

\section{BOULDER, COLO.}

Cryogenic Engineering Laboratory. Cryogenic Equipment. Cryogenic Processes. Properties of Materials. Cryogenic Technical Services.

\section{CENTRAL RADIO PROPAGATION LABORATORY}

Ionosphere Research and Propagation. Low Frequency and Very Low Frequency Research. Ionosphere Research. Prediction Services. Sun-Earth Relationships. Field Engineering. Radio Warning Services. Vertical Soundings Research.

Radio Propagation Engineering. Data Reduction Instrumentation. Radio Noise. Tropospheric Measurements. Tropospheric Analysis. Propagation-Terrain Effects. Radio-Meteorology. Lower Atmosphere Physics.

Radio Systems. Applied Electromagnetic Theory. High Frequency and Very High Frequency Research. Modulation Research. Antenna Research. Navigation Systems.

Upper Atmosphere and Space Physics. Upper Atmosphere and Plasma Physics. Ionosphere and Exosphere Scatter. Airglow and Aurora. Ionospheric Radio Astronomy.

\section{RADIO STANDARDS LABORATORY}

Radio Physics. Radio Broadcast Service. Radio and Microwave Materials. Atomic Frequency and TimeInterval Standards. Millimeter-Wave Research.

Circuit Standards. High Frequency Flectrical Standards. Microwave Circuit Standards. Electronic Calibration Center. 
